\title{
De Carl Schmitt à Christian Meier
}

Les Euménides d'Eschyle et le concept de «politique » (« das Politische »)

\section{Paul Demont}

\section{(2) OpenEdition}

1 Journals

\section{Édition électronique}

URL : http://journals.openedition.org/philosant/1076

DOI : 10.4000/philosant.1076

ISSN : 2648-2789

\section{Éditeur}

Presses universitaires du Septentrion

\section{Édition imprimée}

Date de publication : 1 novembre 2011

Pagination : 151-172

ISBN : 978-2-7574-0356-3

ISSN : 1634-4561

\section{Référence électronique}

Paul Demont, « De Carl Schmitt à Christian Meier », Philosophie antique [En ligne], 11 | 2011, mis en

ligne le 01 novembre 2018, consulté le 30 avril 2019. URL : http://journals.openedition.org/

philosant/1076 ; DOI : 10.4000/philosant.1076

\section{(c) (i) (9)}

La revue Philosophie antique est mise à disposition selon les termes de la Licence Creative Commons Attribution - Pas d'Utilisation Commerciale - Pas de Modification 4.0 International. 


\section{DE CARL SCHMITT À CHRISTIAN MEIER \\ Les Euménides d'Eschyle et le concept de «politique» (« das Politische »)* \\ Paul Demont \\ Université Paris IV Paris-Sorbonne}

RESUME : Dans La naissance du politique (Paris, 1995), Christian Meier propose, à partir de l'étude des Euménides d'Eschyle, une réinterprétation et une correction en un sens démocratique du « concept du politique » élaboré par Carl Schmitt.

SUMMARY: Christian Meier's analysis of Aeschylus's Eumenides in Die Entstehung des Politischen bei den Griechen (Frankfurt am Main, 1980) investigates a democratic version of Carl Schmitt's theory of Politics.

\footnotetext{
* Cette étude est une version entièrement refondue de « Christian Meier, Carl Schmitt, amis, ennemis et politique dans Les Euménides d'Eschyle », dans J. Peigney (éd.), Amis et ennemis en Grèce ancienne, Bordeaux, Ausonius, 2011, p. 57-68. Je remercie Jocelyne Peigney de m'avoir autorisé à reproduire une partie des analyses présentées à Tours. J'ai bénéficié ensuite de remarques précieuses de Hinnerk Bruhns et de J. Harvey Lomax. J'exprime enfin ma profonde reconnaisssance au Professeur Christian Meier, qui a bien voulu lire la première version de cette étude et y réagir dans une lettre du 7 mai 2010 (Meier, perlitt. 2010).
}

Philosophie antique, $\mathrm{n}^{\circ} 11$ (2011), 151-172 

Le livre de Christian Meier qui a été traduit en français sous le titre $\mathrm{La}$ naissance du politique en 1995 est paru en 1980 à Francfort sous le titre Die Entstehung des Politischen bei den Griechen ${ }^{1}$. C'est à Carl Schmitt que Christian Meier emprunte ce concept $d u$ politique («das Politische »), comme il l'explique dès l'introduction, et le chapitre V, intitulé «Les Euménides d'Eschyle et l'avènement du politique $»^{2}$, est dédié Carolo

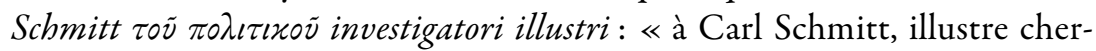
cheur du Politique ». Christian Meier a de plus consacré à Carl Schmitt une étude spécifique, qui mentionne à plusieurs reprises des conversations orales et des échanges de lettres entre les deux hommes dans les années 1968-1975³. Cette référence explicite, mais complexe, mérite d'être étudiée, car elle est passée quasiment inaperçue lors de la traduction du livre en français, et n'est quasiment pas examinée non plus dans un volume récemment consacré à Christian Meier, où il n'en est fait mention ni dans l'étude sur «l'anthropologie du politique », ni dans celle qui est consacrée aux rapports entre tragédie et politique ${ }^{4}$. Dans la réponse de Christian Meier aux intervenants, Carl Schmitt n'est mentionné qu'en passant, dans une

1. Meier 1980, 1995.

2. «Aischylos'Eumeniden und das Aufkommen des Politischen ».

3. Meier 1988, p. 537-566 (voir aussi p. 605-607).

4. Martin 2008, Seidensticker 2008. Signalons que le rapport de l'étude de Christian Meier à Carl Schmitt n'est pas mentionné non plus dans les interventions présentées récemment aux Entretiens de la Fondation Hardt sur Eschyle (Jouanna \& Montanari 2009). Sur les relations entre les deux hommes, «Christian Meier zählt wie (...) Ernst Wolfgang Böckenförde und (...) Reinhart Koselleck (...) zu jener konservativen Geisteselite, für die Carl Schmitts Wohnort Plettenberg die eigentliche geistige Hauptstadt der Bundesrepublik war. Wer nach Plettenberg pilgerte, bekam von Carl Schmitt ein Zimmer in 'Deutschen Haus' reserviert und 'Hausaufgaben' über Nacht gestellt » (Tageszeitung, 15. 03. 2006, référence que je dois à J. Harvey Lomax, traducteur de Meier, H. 1990 en américain). 
liste d'auteurs qui ont pu l'influencer d'une façon ou d'une autre, et auxquels il exprime sa reconnaissance 5 .

Peut-être faut-il rappeler d'abord, de façon schématique ${ }^{6}$, deux aspects de la pensée de Carl Schmitt, à partir de La notion de politique, traduit en français en 1972 (d'après la réédition de Der Begriff des Politischen de 1963, avec une préface et des corollaires ajoutés au texte de 1932) ${ }^{7}$, d'un petit livre intitulé Les trois types de pensée juridique, publié en 1934 et traduit en français en $1995^{8}$, enfin de ses deux ouvrages intitulés Théologie politique, traduits en français en 1988 (le premier, dont la première édition date de 1922 est traduit dans le texte de la réédition de 1934, et la première édition du second date de 1970). Ces aspects sont réunis, à mon avis, dans la formule suivante: «L'État, unité essentiellement politique, dispose du jus belli, c'est-à-dire de la possibilité effective de désigner l'ennemi, le cas échéant, par une décision qui lui soit propre, et de le combattre9 . »

Le premier aspect tient au concept de «décision » et à la théorie du « décisionnisme ». Carl Schmitt emprunte ${ }^{10}$ à Thomas Hobbes un adage « dont la reprise incantatoire tout au long de son œuvre fera l'axiome de principe du décisionnisme $\gg^{11}$ : auctoritas, non veritas, facit legem. Le politique, c'est avant tout la reconnaissance par une société donnée d'une

5. Bernett, Nippel \& Winterling 2008, p. 303 («sei es Machiavelli und Guicciardini, Montesquieu, Tocqueville, Jacob Burckhardt, Otto Hintze oder Carl Schmitt, Norbert Elias, Hannah Arendt, auch Sternberg und Gehlen »). Suit une autre liste de noms qui ne doivent pas être omis, comprenant Otto Vosler, Alfred Heuß, Reinhart Kosellek, «ebensowenig Paul Veyne und Jean-Pierre Vernant $\gg$.

6. On pourra compléter cette présentation sommaire en lisant l'étude de Christian Meier citée note 3 ci-dessus, ou encore, notamment, Meier, H. 1990, Lomax 2000. Le Professeur Meier me signale la parution de Mehring 2009 dont il a rédigé un compte rendu (Meier Chr. 2010b).

7. L'étude Der Begriff des Politischen (pour la version française du titre, on peut préférer à « la notion de politique » la traduction plus précise «le concept du politique »: Zarka 2009, p. 9) est réunie avec une autre étude que l’auteur avait présentée peu auparavant dans l'Espagne franquiste, Théorie du partisan, dans Schmitt 1972. Je cite aussi l'édition allemande de 1932 (qui est la publication revue d'une étude de 1923). Comme on le verra infra, il est possible que la réédition allemande de 1963 ait joué un rôle important à la source même du livre de Christian Meier de 1980.

8. Schmitt 1995.

9. Schmitt 1972, p. 86.

10. On a souvent critiqué l'usage que Schmitt fait de Hobbes. Voir par exemple, outre Leo Strauss (voir H. Meier 1990), Tuchscherer 2004 et Zarka 2009, qui montre que pour Hobbes la souveraineté ne se définit ni par la dictature ni par l'exception, et qu'il y a chez lui une « ontologie de l'individuel » absente de la pensée de Carl Schmitt (« Le mythe contre la raison: Carl Schmitt ou la triple trahison de Hobbes », p. 47-70).

11. Tuchscherer 2004, p. 280. 
instance de décision souveraine de nature « dictatoriale », car non soumise à une exigence de contenu éthique ou de véridicité. « C'est, dit-il, la décision qui fonde la norme et l'ordre. La décision souveraine est commencement absolu, et le commencement (y compris au sens d'arkhè) n'est rien d'autre qu'une décision souveraine. Elle jaillit d'un désordre normatif et d'un désordre concret ${ }^{12}$. » Cette capacité de prendre une décision dans un contexte d'urgence pour maintenir un «ordre », fût-il « un ordre qui n'est pas de droit », est, selon Carl Schmitt, ce qui fonde proprement la souveraineté : «Est souverain celui qui décide de la situation exceptionnelle », selon la première phrase de la Théologie politique de $1934^{13}$. Le second aspect est lié au premier. La décision politique la plus caractéristique $d u$ politique, c'est la décision de faire la guerre contre un ennemi. Ainsi « les peuples se regroupent conformément à l'opposition ami-ennemi » et « cette opposition subsiste à l'état de virtualité réelle pour tout peuple qui a une existence politique $\gg^{14}$. Carl Schmitt précise que, par «ami » et « ennemi », il ne s'agit pas d'entendre une métaphore, ni un sentiment individuel et privé, que les mots ne désignent ni un simple «concurrent » dans la rivalité économique qu'instaure le libéralisme, encore moins un partenaire ou un adversaire dans une discussion. « L'ennemi ne saurait être que l'ennemi public (...) hostis et non inimicus au sens le plus large; $\pi 0 \lambda \dot{\varepsilon} \mu 10$ et non $\dot{x} \chi \theta p_{0} \varsigma^{15}$. » Donc l'ennemi est celui qui est désigné comme tel par la décision souveraine de l'État, et cette désignation est ce qui fonde la communauté politique. Analyse terrible et bien souvent confirmée par les faits. En note, Carl Schmitt, avant de citer le Lexicon totius Latinitatis de Forcellini, mentionne la République de Platon $(\mathrm{V}, 470)$ : «Platon souligne fortement l'opposition (...), mais en la mettant en rapport avec cette autre opposition entre $\pi \dot{\partial} \lambda \varepsilon \mu \circ \varsigma$ (la guerre) et $\sigma \tau \dot{\alpha} \sigma \varsigma \varsigma$ (émeute, soulèvement, rébellion, guerre civile). Aux yeux de Platon, seule une guerre entre Grecs et Barbares (« ennemis de nature ») est une guerre véritable, alors que les luttes entre les Grecs sont des $\sigma \tau \dot{\alpha} \sigma \varepsilon ı \zeta$ (des querelles intestines). L'idée qui domine ici

12. Schmitt 1995 , p. 83 , commenté par Tuchscherer 2004, p. 281-282, qui analyse les difficultés de cette doctrine chez Hobbes lui-même et les nombreuses «inflexions » que Schmitt lui fait subir.

13. Schmitt 1988, p. 15, avec une note importante du traducteur (la phrase allemande est : «Souverän ist, wer über den Ausnabmezustand entscheidet 》). Voir aussi p. $22: \ll \mathrm{Il}$ subsiste malgré tout un ordre, fût-ce un ordre qui n'est pas de droit. L'existence de l'État garde ici une incontestable supériorité sur la validité de la norme juridique (...) L'État suspend le droit en vertu d'un droit d'autoconservation, comme on dit. »

14. Schmitt 1972, p. 69.

15. Ibid. 
est qu'un peuple ne peut se faire la guerre à lui-même et qu'une guerre civile n'est jamais qu'autodestruction et ne saurait signifier la naissance d'un État nouveau, voire d'un peuple nouveau. »

Or c'est en grande partie dans cette perspective, mais en la transformant explicitement d'une façon décisive, que Christian Meier lit Les Euménides, pièce où, dit-il, «le politique s'est exprimé avec le plus de justesse, alors même qu'il advenait à Athènes $»^{16}$. Rappelons que la pièce d'Eschyle, qui conclut la trilogie de L'Orestie, après Agamemnon et Les Choéphores, met en scène une solution inédite permettant à l'Argien Oreste, fils d'Agamemnon et de Clytemnestre, frère d'Électre, poursuivi par les Érinyes, ces Furies attachées à la vengeance du sang maternel, d'être débarrassé du poids de son crime. Cette solution est juridique : l'acquittement d'Oreste par le jugement, à Athènes, du Conseil de l'Aréopage, qu'institue la déesse Athéna dans la deuxième partie de la pièce pour juger les crimes de sang, et qui prend cette décision devant les spectateurs par un vote à la majorité, à une voix près, celle d'Athéna, après l'intervention d'Apollon pour défendre Oreste. Rappelons aussi que la trilogie fut représentée en 458, quatre ans après une réforme très importante de la démocratie athénienne, dans laquelle Éphialte avait retiré au Conseil de l'Aréopage (formé des anciens Archontes) tous ses pouvoirs politiques, pour les donner principalement au Conseil des Cinq Cents (formé de cinquante citoyens par tribu, tirés au sort pour un an $)^{17}$.

Carl Schmitt avait voulu redéfinir le politique face à sa «neutralisation » libérale sous diverses formes, dans ce qu'on appelle l'entre-deuxguerres, selon une expression qui prend ici un sens terrible; dans le contexte de l'Allemagne des années 1970-1980, Christian Meier veut à nouveau restaurer, au moyen de l'analyse de l'Athènes archaïque et classique, «le sens du politique » qui tend, regrette-t-il, à se réduire à « une simple fonction de l'économie ${ }^{18}$. Mais s'il rend hommage au juriste, il précise

16. Meier 1995, p. $107=$ Meier 1980, p. $144: \ll$ In ibm ist das Politische in dem Moment zur Sprache gebracht worden, in dem es sich in Athen zum ersten Mal und in erschütternder Weise voll verwirklichte. Und dies in einer unerbört adäquaten, noch heute aktuellen Weise. 》

17. «Éphialte (...) écarta beaucoup d'Aréopagites (...), ensuite, sous l'archontat de Conon, il dépouilla le conseil de tous les pouvoirs supplémentaires grâce auxquels il était le gardien du régime, et remit les uns aux Cinq Cents, les autres au peuple et aux tribunaux (...). Éphialte périt assassiné peu après. » (Aristote, Constitution d'Athènes, 25, trad. M. Sève, Paris, Le Livre de Poche, 2006.)

18. Meier 1995, p. 13 (= Meier 1980, p. 14 : «der Sinn für das Politische [...] eine Funktion des Wirtschaftlichen $\gg)$. 
immédiatement qu'en grec, «le politique » implique au contraire «le dépassement » de la discrimination de l'ami et de l'ennemi ${ }^{19}$. Il lui faut donc en quelque sorte récrire Carl Schmitt, presque à l'envers, et c'est pourquoi, peut-on penser, la première partie de son livre, qui définit la formation du politique en Grèce (bei den Griechen, dit le titre allemand, aspect malheureusement supprimé dans le titre français), reprend quasiment le titre de Carl Schmitt et $s^{\prime}$ intitule «Le concept de politique ${ }^{20}$. Il y a donc un aspect paradoxal dans cette entreprise, qui, en quelque sorte, cherche à démocratiser, mais au sens antique, une analyse conçue largement contre la démocratie libérale bourgeoise de la «clasa discutidora $\gg^{21}$.

Dès le début du livre apparaît une première caractéristique de cette réécriture, qui consiste à remplacer la notion d'État, impropre pour le monde antique et même pour une bonne part du monde moderne (et que Carl Schmitt lui-même avait peu à peu récusée pour la remplacer par celle de « peuple », puis de « partisans $»^{22}$ ), par celle de communauté des citoyens dans la polis ${ }^{23}$. Il faut dès lors, selon Christian Meier, distinguer la question de l'unité politique et le politique en lui-même, qui suppose seulement « un certain degré d'association et de dissociation », selon une formule de Carl Schmitt que cite Christian Meier ${ }^{24}$. En tant que tel, le politique peut ainsi être disjoint de la discrimination de l'ami et de l'ennemi. C'est ce qui conduit à une belle analyse des réformes de Clisthène en termes d'institutionnalisation de ce que Meier appelle la «présence civique » à Athènes, selon une expression reprise probablement de Hannah $\mathrm{Arendt}^{25}$, à laquelle

19. Meier 1995, p. 14 (= Meier 1980, p. 16 : «die Überwindung diesen Gegensätze »).

20. Ou plutôt : «Zum Begriff des Politischen ». L'écart est signifiant, Meier écrivant «à propos du "concept du politique" » (scil. tel qu'il a été défini par Carl Schmitt).

21. Expression de Donoso Cortés qu'affectionne Carl Schmitt (par ex. Schmitt 1988, p. 67).

22. Cf. Trierweiler 2009, p. 27.

23. Cf. la première phrase du premier chapitre: «Les Grecs appelaient politique ce qui était propre à la polis» (Meier 1995, p. 25): «wenn die Griechen das der Polis Eigene politisch nannten, so... » (Meier 1980, p. 27). L'emploi du mot remonte principalement à Jacob Burckhardt (cité à la fois par Schmitt et par Meier) : cf. Bauer 2001, p. 119. L'adjectif

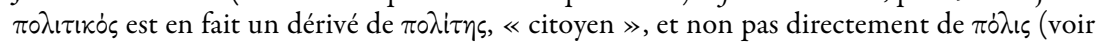
Demont à paraître).

24. Meier 1995, p. 31 (= Meier 1980, p. 36 : «einen bestimmten Intensitätsgrad von Assoziation und Dissoziation $\gg)$.

25. «Der bürgerlichen Gegenwärtigkeit» (p.91), expression que Meier cite aussi en français dans son texte allemand : ce chapitre a été présenté à Paris dès 1972 et publié une première fois en 1973 (Meier 1973). Les analyses de Hannah Arendt réhabilitant la praxis contre le «yankisme » présentent de fait des aspects assez comparables (voir une présenta- 
il donne un contenu beaucoup plus concret et historique : ces réformes aboutissent effectivement à rendre chaque citoyen, en association avec les membres de son dème, de sa trittye et de sa tribu, présent, à un moment ou à un autre, au centre de la décision politique. Cet aspect n'est pas ce qui nous occupe aujourd'hui. En revanche, une fois la notion d'État déclarée, avec raison, impropre à l'étude de la Grèce antique, que devient la notion de décision souveraine, quelle est la nature de cette décision politique que je viens de mentionner ? Christian Meier, dans son chapitre sur Clisthène, va jusqu'à écrire que, dans la nouvelle configuration introduite par le réformateur, le «contenu » de la décision était souvent «moins intéressant pour les citoyens que leur participation à la décision $»^{26}$. Les concepts de « décision » et de «situation exceptionnelle », si importants dans la théorie, ou le mythe, schmittiens ont-ils encore une pertinence ? Si le chapitre qui suit immédiatement, et qui est consacré aux Euménides, est dédié à Carl Schmitt, c'est peut-être parce qu'il tente de répondre à cette question. Christian Meier y construit en effet, à partir de la pièce d'Eschyle ${ }^{27}$, une sorte de modèle démocratique paradoxal $\mathrm{du}$ « décisionnisme » schmittien, que je voudrais décrire, avant de montrer comment, néanmoins, la problématique de l'ami et de l'ennemi est aussi introduite parallèlement dans son analyse.

On a souvent observé que l'affrontement des droits d'Oreste et de Clytemnestre, par le biais de l'affrontement d'Apollon et des Érinyes, est un combat où aucun des deux camps n'a une supériorité religieuse, morale ou intellectuelle manifeste. Dès lors, comment trancher un tel débat entre humains, mais aussi, et surtout, entre dieux ? Pour Meier, il ne faut pas trop chercher à savoir dans quel sens Eschyle penche, mais plutôt observer que la pièce pose précisément cette question de la décision. La décision pourrait revenir à Athéna, comme les deux parties le lui demandent expressément, et ce serait alors une image de la décision de type dictatorial, mais la déesse décide de la déléguer au Conseil de l'Aréopage, qu'elle fonde pour l'occa-

tion générale dans Demont 2002). Chr. Meier a correspondu une fois avec Hannah Arendt («leider nur [...] ein einiges Mal », Meier 2010 per litt.).

26. Meier 1995, p. 95.

27. Notons un écart entre le titre allemand du sous-chapitre («Die Eumeniden als Zeugnis frühen politischen Denkens », p. 177) et la traduction française («Les Euménides comme témoignage d'une pensée politique nouvelle », ibid., p. 125). La traduction perd l'idée que, pour Meier, la pensée politique, prise au début, n'obéissait pas à la problématique schmittienne. Le Professeur Meier me précise que le travail sur Les Euménides a été effectué à partir de 1977 pour deux conférences à Berlin, puis rédigé au début de 1979 à Princeton, avant les chapitres sur la notion de politique. 
sion. Et celui-ci, après avoir entendu les deux parties, décide par un vote. De la même façon, Meier transpose l'analyse de la décision politique de la dictature à la démocratie. Meier insiste sur le caractère exceptionnel de la mise en scène de cette « problématique de la décision ${ }^{28}$ : les Aréopagites votent à tour de rôle devant le public, et il se produit « cette chose inouïe, qui paraît presque absurde $\gg^{29}$ : «la décision (...) se fait avec le minimum de voix, et qui plus est, elle est prise contre la majorité des Aréopagites », puisqu'il faut qu'Athéna vote en faveur d'Oreste pour que les voix soient en nombre égal, égalité dont Athéna a dit auparavant qu'elle profiterait à l'accusé ${ }^{30}$. Meier commente : «Ce qu'entrevoit cette réflexion radicale sur la décision problématique de 462/461, c'est in nuce le décisionnisme. La construction audacieuse du vote indique précisément la conscience de devoir séparer validité et vérité dans le processus politique de la décision. » Les réformes d'Éphialte sont décrites en termes de « décision problématique », et correspondent dans une large mesure à la définition schmittienne de la décision exceptionnelle, et même à son vocabulaire, et elles sont projetées sur la décision de l'Aréopage des Euménides, qui leur donne un cadre interprétatif nouveau, toujours schmittien, mais dans la perspective du vote majoritaire après un débat. La mise en scène, tout à fait exceptionnelle dans le théâtre grec antique, et même dans l'histoire générale du théâtre, d'un vote nominal, transforme la décision révolutionnaire en processus régulier : «Elle montre que c'est en fin de compte toujours la majorité qui décide et ce, quelle que soit la validité des raisons qui motivent la décision de chacun ; elle affirme enfin que cette décision est un lien, sans pour autant être issue d'arguments convaincants ${ }^{31}$. $\gg$ Autrement dit, l'avènement du politique schmittien est observable à Athènes, en cette année 458 avant notre ère, dans une pièce de théâtre, donc dans une construction littéraire et

28. « Die Problematik der Entscheidung (Meier 1980, p. 190).

29. «Und da findet sich das Unerhörte, geradezu absurd Anmutende », ibid. p. 191.

30. Cela suppose qu'Athéna mette son suffrage avant le décompte des voix, ce que le texte semble bien impliquer (v. 734-735), en annonçant que l'égalité vaudra acquittement (annonce qui sert d'explication à la pratique athénienne). Cf. le résumé des discussions par Sommerstein 1989, p. 211-226.

31. Meier 1995, p. 135-136 (= Meier 1980, p. $194:$ : Es ist in nuce die Einsicht des Dezisionismus. Gerade die kühne Konstruktion des Abstimmung weist auf das Bewußtsein, daß Geltung und Wabrheit im politischen Entscheidungsproceß zu trennen sind; daß, wie begründet auch immer der Einzelne zu seinem Votum kommt, am Ende die Mehrheit entscheidet; daß diese Entscheidung verbindlich ist, ohne deswegen zwingenden Argumenten $z u$ entspringen. ») On pourra comparer : «Considérée d'un point de vue normatif, la décision est née d'un néant. La force juridique de la décision est autre chose que le résultat des arguments qui la fondent. » (Schmitt 1988, p. 42.) 
artistique consciente: il s'agit bien du concept du politique. Mais ce concept du politique repose sur la persuasion, des citoyens, et des Érinyes. «Comme le dit Athéna, la victoire de la Peithô est la victoire de Zeus en tant qu'il est le protecteur divin de l'Agora ${ }^{32}$. [...] La persuasion représentait la solution pacifique, politique, et pour cette raison il fallait toujours la préférer à l'opposition violente. Cela présupposait que l'on conçoive le citoyen dans son entière présence. Il s'agit là justement de l'un des traits du politique chez les Grecs ${ }^{33}$. » Donc, le politique, mais le politique grec, qui, après tout, ne semble pas sans rapport avec les procédures de la «clasa discutidora » vitupérée par Carl Schmitt.

Néanmoins, Christian Meier fait aussi la place à l'autre aspect du politique schmittien, la différenciation de l'ami et de l'ennemi, dont il a dit dès le début de son ouvrage qu'elle n'était que la « forme extrême, qui n'est pas nécessairement la plus politique $\gg$ du concept de politique ${ }^{34}$, car il l'observe aussi, de façon éclatante, dans Les Euménides.

En bon historien, et à la différence de trop de philologues, il décrit le contexte immédiat de L'Orestie, les réformes, puis l'assassinat d'Éphialte, après la «destitution de l'Aréopage ». Ce contexte historique est celui de la constitution de la puissance athénienne contre Sparte et de la puissance du peuple contre les nobles. Meier est ici un politologue disciple fidèle de Schmitt, notamment lorsqu'il insiste sur le sens uniquement politique à donner à cette relation d'hostilité : « l'ennemi était seulement l'autre, celui qui menaçait l'existence de quiconque, il n'était absolument pas idéologiquement disqualifié $e^{35}$. » Dans une longue note, Christian Meier insiste sur « la représentation grecque des guerres », qu'il construit à partir d'Homère et d'Hérodote: « elle traite les ennemis sans hostilité particulière et les considère comme fondamentalement égaux (y compris les femmes, les Amazones) ». On peut voir dans cette généralisation et cette idéalisation ${ }^{36}$

32. Cf. v. 974 .

33. Meier 1995, p. 138 (= Meier 1980, p. 196 : «Voraussetzung war, daß man die Bürger in ihrer Gegenwärtigkeit für voll nahm. Eben das gehört zum Politischen der Griechen. »)

34. Meier 1995, p. 31.

35. Ibid. p. 111 ((= Meier 1980, p. 152 : « der Feind war nur der andere, der die eigene Existenz bedrohte, in keiner Weise ideologisch disqualifiziert 》), avec la note 20 p. 377 (= Meier 1980, n. 26 p. 152).

36. Le Professeur Meier conteste cette appréciation, en précisant que son analyse a pour origine la comparaison répétée des représentations de l'ennemi sur les sculptures du British Museum, de même taille sur les métopes de Bassae, d'une taille beaucoup plus petite sur les œuvres orientales («Immer wieder bin ich an den Metopen von Bassai vorbeigegangen, immerwieder an den Darstellungen des orientalischen Monarchien, denen gegenüber die Feinde 
une première trace de la conception qu'a Schmitt du rôle de l'affrontement ami/ennemi dans la constitution des peuples.

Quand il passe de la reconstitution du contexte historique à l'analyse de la pièce d'Eschyle, Christian Meier n'étudie pas le vocabulaire de l'ami et de l'ennemi dans Les Euménides en général, mais se focalise sur un emploi remarquable de la fin de la pièce. Les emplois précédents pourraient pourtant appuyer son analyse du peu de validité éthique ou rationnelle de la décision finale, et ils installent de fait une relation ami/ennemi au cœur de la pièce. En effet, le vocabulaire de la philia est utilisé régulièrement par Clytemnestre et par les Érinyes pour désigner la relation mère/fils (v. 100, 271, 356,608 , et peut-être $119^{37}$ ), Oreste l'emploie pour la relation père/fils (v. 608), tandis qu'Apollon l'utilise, lui, pour la relation conjugale (v. 216). Ces emplois, tous justifiés, conduiraient à une condamnation générale des crimes commis, non à l'acquittement d'Oreste. D'autre part, Apollon est le seul à utiliser le vocabulaire de l'echtra: il le fait à propos des Érinyes considérées comme les « ennemies » d'Oreste (v. 66 et 730). Le v. 730, en particulier, appartient au dernier des dix couples de vers qui accompagnent le vote des Aréopagites, au moment décisif où vont intervenir les deux derniers votes. Mais Meier ne s'intéresse qu'à un autre emploi, ultérieur, celui du vers 988, qui est de fait assez exceptionnel, dans le chant final des Euménides qui appelle les Athéniens à repousser la stasis (le mot est employé),

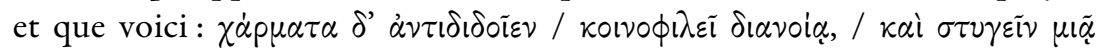

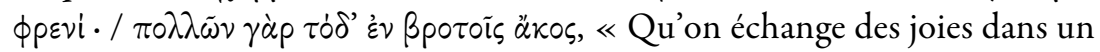
esprit commun d'amour et qu'on haïsse d'un seul cœur; voilà chez les humains le remède à bien des maux » (v. 984-987, trad. personnelle) ${ }^{38}$.

ganz klein waren, während in Bassai von gleich zu gleich gekämpft wurde. Gewiß ist es richtig, daß zwischen Griechen und Barbaren, Griechen und Amazonen eine Asymmetrie besteht. Aber - bis auf das berühmte Vasenbild nach der Schlacht am Eurymedon- begegnet [man] nicht die Verachtung, nicht die Geringschätzung des Gegners. Das ist gemeint - und da wäre in der Tat eine Entsprechung zu Carl Schmitts Bemerkung, obwohl er ganz anderes im Auge hat als ich », Meier 2010 per litt.).

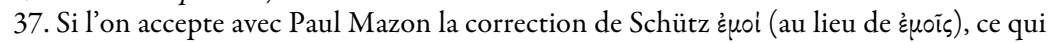
n'est pas très facile à comprendre cependant ( $\ll 119$ has never been satisfactorily interpreted or emended $\gg$, note Sommerstein ad loc.).

38. Ibid. p. 146-147 avec p. 389 n. 169. Dans la version française du livre de Meier: «Qu'on échange des joies dans un commun amour et qu'on se haïsse d'une seule âme : c'est un grand remède chez les humains », il faut corriger «qu'on se haïsse en «qu'on haïsse », mais le texte allemand est un peu différent : « Freuden möge man sich wechselseitig geben, in gemeinfreundschaftlichem / das Allgemeine liebendem Geiste (Droysen : Eins in der Liebe zum Ganzen), und hassen mit einem Sinn. Vieler Übel unter den Sterblichen ist das das Heilmittel.» (Meier 1980, p. 208.) Meier y juxtapose deux interprétations possibles de 
Il introduit ce passage en rappelant les paroles antérieures d'Athéna aux Érinyes, aux vers 858-866, qui forment un ensemble souvent jugé interpolé depuis Dindorf, car il rompt l'équilibre des répliques, mais qui correspond exactement au contexte historique de la pièce, et dont on ne voit pas comment expliquer l'interpolation si Eschyle n'en est pas l'auteur ${ }^{39}$. Athéna ordonne aux Érinyes de ne pas instiller dans les entrailles et le cœur des Athéniens « Arès [c'est-à-dire le désir de meurtre] pour le mettre au sein de leur peuple et les lancer avec fougue les uns contre les autres $\gg$. Non, dit-

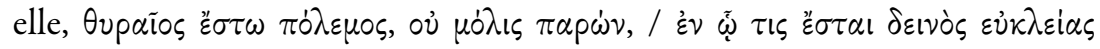

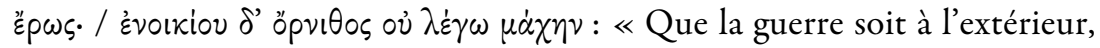
et elle est là aisément ${ }^{40}$, pour celui en qui se trouve un formidable désir de la gloire, mais je rejette le combat avec un oiseau de la même maison ${ }^{41}$ ! »

C'est seulement ici, au vers 864 , qu'intervient dans la pièce le mot $\pi \dot{\partial} \lambda \varepsilon$ -

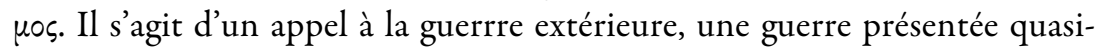
ment comme fraîche et joyeuse, « an astonishing phrase, implying a frank, unashamed, almost cheerful militarism », commente A. Sommerstein ${ }^{42}$. Il est bien compréhensible de commenter le chant ultérieur des Euménides, qui peut avoir les mêmes connotations bellicistes, en relation avec ce passage. Mais le commentaire, tout en s'appliquant assez bien au texte grec ${ }^{43}$, paraît alors directement inspiré des analyses de Carl Schmitt, tout en y ajoutant les innovations propres à l'auteur : «Amitié (philia) vers l'intérieur, unanime hostilité vers l'extérieur. (...) L'inimitié ne doit plus se porter vers l'intérieur, mais résolument vers le dehors : c'est, en rapport avec la polis, un genre nouveau de distinction ami/ennemi qui doit se faire, une réarticulation de la constellation ami/ennemi. C'est ainsi que la polis doit

l'adjectif koinophiles, qu'il développe p. 211 de l'édition allemande, p. 148 de l'édition française : «C'est la koinophilès dianoia dans sa double signification d'amour du tout (du koinon) et d'amitié commune à tous », en renvoyant pour la première à Droysen.

39. Il a pu être ajouté par Eschyle lui-même à un stade quelconque de la publication de la pièce : $c f$. Sommerstein (suivant K. J. Dover), p. 251-252.

40. Sommerstein 1989 , p. 30, va un peu trop loin quand il propose la traduction: « May you have external war, and plenty of it ».

41. Traduction personnelle.

42. Ad loc. opposant, peut-être avec trop d'optimisme, «the normal Greek attitude », exprimée dans un adage fameux d'Hérodote (VIII, 3.1).

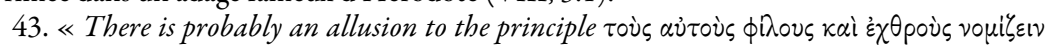
which was often a requirement in treaties of alliance between states (e.g. IG $I^{3}$ 89.28) and a fortiori was vital for civic peace within a state », commente fort bien Sommerstein. Si Carl Schmitt a connu cette expression grecque, il a pu l'utiliser à l'appui de ses théories. 


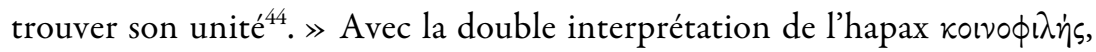
l'amitié, «pour la première fois », en tant qu'élan commun de haine et d'amitié et élan vers la totalité, devient politique.

Faisons une brève parenthèse. Meier ajoute une note où il cite Platon : «Par la suite, Platon fera, dans les grandes lignes, une distinction analogue entre ami et ennemi », puis il discute les sens de echthros et de polemios. Il semble bien qu'il parte ici de la note de Carl Schmitt dans La notion de politique que j'ai citée au début de mon analyse ${ }^{45}$. Mais Meier ajoute un correctif important, en citant des exemples qui contredisent et ce passage de Platon et Carl Schmitt (notamment Agamemnon, v. 608 et plusieurs passages de Platon lui-même), en renvoyant à une étude grecque de Ion X. Contiades de 1969 et en annonçant un travail en cours de Peter Spahn ${ }^{46}$. Il en conclut : «Le rapport entre les deux termes est difficile à éclaircir. Les champs de signification se recouvrent (...) Polemios a son centre de gravité dans l'antagonisme immédiat, caractéristique de la guerre, alors que echthros est plus général. Il est le seul à servir à la construction d'un concept de l'inimitié (echthra). » Autrement dit, et cela est tout à fait exact, le vocabulaire grec ne connaît pas la distinction tranchée que veut y voir Carl Schmitt.

Cela dit, Christian Meier peut tout de même lire la fin des Euménides dans l'esprit de Carl Schmitt. Il le peut d'autant mieux qu'il introduit ici, en tant qu'historien, une inimitié véritable, contre Sparte, que peuvent évoquer les allusions à la guerre en général (v. 776-777, 913-915, 1009) et l'alliance athénienne avec Argos, qu'évoque incontestablement l'accueil d'Oreste l'Argien à Athènes, et qui est mentionnée plus ou moins allusivement trois fois dans la pièce (v. 289-291, 669-673, 762-764). Ainsi, le décisionnisme démocratique est tout de même lié à la construction d'Athènes comme communauté politique dans le cadre de la discrimination entre l'ami et l'ennemi. La reconstruction historique et la fidélité aux analyses de

44. Meier 1995, p. 147 (= Meier 1980, p. 208-209: Freundschaft (philia) also nach Innen, einmütige Feindschaft nach Außen (...) Feindschaft soll nicht mehr nach Innen, dafür geschlossen nach Außen sein: eine neue Polis-bezogene Scheidung von Freund und Feind soll stattfinden, eine Verlagerung der Freund-Feind Konstellation. Darin soll die Polis ibre Einheit bewinnen »).

45. Voir supra p. 155. Cela apparaît plus encore si l'on tient compte d'une phrase omise dans la traduction française : «Platon hat nachher im Großen eine analoge Freund-FeindScheidung getroffen. (...) Platon unterscheidet entsprechend zwischen polemos gegen die Barbaren und stasis (Zwietracht, Bürgerkrieg unter den Griechen), s. Politeia $469 \mathrm{f}$. Vgl. Menexenos 245c. ( (Meier 1980, p. 208.)

46. A-t-il été mené à terme ? Il ne semble pas. 
Carl Schmitt vont de pair : «Le clivage ami/ennemi est encore à cette époque une catégorie vivace dans la pensée des nobles, et c'est de là qu'Eschyle est parti ${ }^{47}$. » Ce clivage s'est ajouté à un clivage, dans les mêmes termes, entre les nobles et le peuple, dont Christian Meier estime qu'il est transposé dans le conflit de L'Orestie. Mais apparaît une nouvelle solidarité, « qui présupposait une hostilité unanime contre l'extérieur », d'abord au sein de la Confédération de Délos, puis, au moment des Euménides, dans l'alliance avec Argos ${ }^{48}$, et qui est associée dans Les Euménides à l'avènement $d u$ politique grec: «conflit et décision, intérieur et extérieur, ami et ennemi, mais aussi et surtout réconciliation et compromis $\gg^{49}$. Voici donc, dans une extraordinaire alliance des contraires, la pratique régulière du « compromis » unie à la décision exceptionnelle dans la naissance du politique en Grèce ${ }^{50}$.

Un autre aspect de l'imprégnation schmittienne, mais surtout de la distance prise par rapport à Schmitt, tient à l'analyse que fait Meier du rôle des dieux dans Les Euménides. Il se réfère explicitement à l'ouvrage de Carl Schmitt publié sous le titre Politische Theologie en 1922 et republié, avec une seconde étude très différente, en $1970^{51}$, quand il donne comme titre du dernier sous-chapitre de son étude de la pièce d'Eschyle : «"Politique théologique". Les Euménides d'Eschyle dans la tradition de la pensée politique grecque ». L'expression mise entre guillemets inverse le titre de Carl Schmitt : Les Euménides proposent selon Meier non pas une théologie politique, mais une politique théologique, « un pendant intéressant à la théologie politique de l'époque moderne et contemporaine $\gg^{52}$.

Une perspective de Carl Schmitt, qui est développée à la fin de la seconde étude de Politische Theologie, aurait pu être alléguée, mais ne l'est pas. Schmitt voit dans un passage de Grégoire de Nazianze sur la doctrine

47. Meier 1995, p. 147 (= Meier 1980, p. 210 : « Das Denken in Freund-Feind-Kategorien blieb auch dann noch unter den Adligen lebendig. Daran knüpft Aischylos an. »)

48. Ibid. p. 148.

49. Ibid. p. 154.

50. On comparera les observations sur le libéralisme que Schmitt emprunte à Donoso Cortés : «Son essence est la négociation, les demi-mesures conservatoires, avec l'espoir que la confrontation définitive, le combat sanglant qui fera la décision, puisse se muer en débats parlementaires et être éternellement remis grâce à une éternelle discussion. La dictature est le contraire de la discussion. » Et il rapproche cela du «système d'un Condorcet, par exemple », où « la société humaine se métamorphose en un immense club » et où, « de la sorte, la vérité se fait de soi grâce au scrutin. » (Schmitt 1988, p. 71.)

51. Traduction française : Schmitt 1988.

52. Meier 1995, p. 157, avec une note qui récuse explicitement, dans le cas de la Grèce antique, la fondation du politique sur des concepts théologiques sécularisés. 
trinitaire, qui décrit la stasis de l'Un contre lui-même ${ }^{53}$, une véritable «stasiologie théologico-politique », capable de fonder une sorte de « récidive gnostique $\gg^{54}$. Le nemo contra deum nisi deus ipse que Goethe a mis en tête du quatrième livre de Dichtung und Wabrheit y est rattaché à l'idée qu' «à toute unité sont immanentes une dualité et par conséquent une possibilité de révolte, une stasis ». Pour Schmitt, «l'homme nouveau » (celui de Hans Blumenberg) est aussi entièrement déthéologisé que dépolitisé, il est «tabula rasa », et n'existe même plus en tant qu'homme, si bien qu'il termine son livre en parodiant de façon sarcastique la sentence de Goethe. Carl Schmitt estime que l'origine de cette sentence se trouve dans les fragments dramatiques de Jakob Michael Lenz sur Catherine de Sienne ${ }^{55}$. Denis Trierweiler observe que le deus contra deum de ces fragments est un emprunt aux Choéphores d'Eschyle ${ }^{56}$, et, de fait, le conflit interne au monde divin, dans L'Orestie, entre les dieux anciens que sont les Érinyes et les divinités nouvelles que sont Apollon et Athéna, est un élément fondamental de l'intrigue. Mais il n'est pas analysable dans une perspective gnostique, et c'est pourquoi Christian Meier l'étudie, non pas dans le cadre de la théologie, mais dans celui du passage violent de l'ancien droit au « nouveau droit » par une décision exceptionnelle.

Christian Meier, lui, place donc le politique à l'origine, sous la protection, « pour la dernière fois $»^{57}$, des dieux, avec la garantie du « milieu » et de «l'ordre juste » qui sont exigés aussi bien par Zeus et Athéna que par les Érinyes. Les dieux, « dans le monde et pas hors de lui, étaient soumis à une légalité plus générale $\gg^{58}$. Dans le monde d'Eschyle, la politique est conçue comme théologique, et l'ordre politique est garanti par la justice divine. L'autonomie du politique naît de la volonté d'Athéna. À la fin du chapitre, ce n'est plus Carl Schmitt qu'évoque Meier, mais son objectif reste le même. Selon Walter Benjamin, à la différence du drame baroque, qui prend pour thèmes l'histoire et la question de la souveraineté comme «art de gouverner », la tragédie d'Eschyle, elle, repose sur le mythe

53. De filio (Or. 29), 2, 7. Schmitt 1988, p. 173-175 (avec une longue note sur le sens du mot stasis, qui contient une étrange remarque sur la pax americana de l'après-guerre).

54. Expression reprise à Blumenberg 1966, son adversaire principal dans cette étude.

55. Schmitt 1988, p. 178.

56. Trierweiler 2009, p. 41.

57. Meier 1995, p. 165 : « Pour la dernière fois, le meson s'inscrivait dans l'horizon de la politique théologique. Plus tard, il sera surtout appréhendé sociologiquement ou au travers de concepts institutionnels mixtes. »

58. Meier 1995, p. 159. 
héroïque et la succession des généalogies divines. D’un côté, monothéisme, politique et État modernes, de l'autre polythéisme et «politique au sens grec » (expression répétée deux fois).

Christian Meier lui-même s'est situé par rapport à Carl Schmitt, huit ans après la publication de son livre, dans une contribution à un ouvrage collectif sur Carl Schmitt - contribution dont l'existence même atteste l'importance du lien entre les deux hommes. Plus nettement encore que dans son livre, il distingue ce qui lui semble contestable et ce qui lui semble utile dans la notion de politique.

Dans une première partie, il brosse un tableau, qui n'est pas sans ironie, de l'entreprise quasi platonicienne de Schmitt, à la recherche, pour la première fois au monde, d'un «concept »du politique ${ }^{59}$. La définition de ce concept est liée d'une part à la notion d'intensité maximale dans la relation d'association entre les hommes, et d'autre part à son apparition dans les situations critiques, exceptionnelles, et particulièrement dans les guerres civiles ou extérieures. Alors apparaît la différenciation entre l'ami et l'ennemi, la « découverte ${ }^{60}$, que Schmitt impose, note Meier, quasiment sans argumentation. Ici, Meier critique en particulier, avec raison, l'absence de toute analyse sémantique historique du mot même de «politique ». Il remarque cependant que ce point est brièvement abordé par Schmitt dans la réédition de $1963^{61}$, et, à ce sujet, il cite une lettre que Carl Schmitt lui a adressée le 29 mai 1968, où il semble vouloir répondre à la critique de Meier, en se référant à une phrase « importante » de Hannah Arendt sur la persistance, en profondeur, de la polis grecque dans les usages du mot « politique ». Il vaut peut-être la peine de citer ce texte, dans lequel Carl Schmitt défend la célèbre première phrase de son livre en assurant au professeur d'histoire ancienne que l'emploi du mot « politique » dans l'expression « le concept

59. « Soweit ich weiß, bezog sich der Gebrauch des substantivierten Adjektivs 'das Politische' vor Carl Schmitt nur auf einzelne Bedeutungen von 'Politischen'. 》 (Meier 1988, p. 540.) La notion de «science politique » est abondamment discutée dans la Grèce classique, comme on le sait (voir quelques réflexions sur ce thème dans Demont à paraître).

60. «Er glaubte sie einfach 'gefunden' zu haben (...) Es gab einen Verblüffungseffekt. Keiner hatte sich bis dabin um den 'Begriff des Politischen' gekümmert. » (Meier 1988, p. 542, qui cite à l'appui les mots d'Ernst Jünger à la parution du livre.)

61. On peut se demander si, comme le suggère aussi une phrase ultérieure de l'article, ce n'est pas cette préface à la réédition de 1963 qui a été l'élément déclenchant du livre de C. Meier (Meier 1988, p. 551). Le Professeur Meier a bien voulu me préciser qu'il ne connaissait pas personnellement Carl Schmitt avant 1967, et que cette hypothèse est donc fragile: «Aber wer weiß das? Nachträglich kann ich das nicht mehr rekonstruiren. » (Meier 2010 per litt.) 
du politique » correspond bel et bien au «mot grec » et que la polis grecque restera toujours « là », aussi longtemps du moins que nous utiliserons encore le mot « la politique ».

Der erste Satz ('der Begriff des Staates setzt den Begriff des Politischen voraus') hat es in sich (also das griechische Wort !). In diesem Zusammenhang ist ein Satz von Hannah Arendt, in der Zeitschrift Merkur Nr. 240 (Seite 313) wichtig :... in der Sprache sitzt das Vergangene unausrottbar (...) Die griechische Polis wird so lange am Grunde unserer politischen Existenz, auf dem Meeresgrunde also, weiter da sein, als wir das Wort 'Politik' im Munde fübren.

La première phrase (« Le concept d'État présuppose le concept de Politique ») a [la notion] en elle (c'est-à-dire, le mot grec!). Une phrase de Hannah Arendt est importante dans ce contexte: « Le passé est inscrit dans le langage d'une façon inextirpable (...) La Polis grecque continuera d'être là au fondement de notre existence politique, comme au fond de la mer, aussi longtemps que nous aurons le mot « Politique » à la bouche.

La référence à Hannah Arendt ${ }^{62}$ est notable, car son influence semble très nette sur Christian Meier lui-même, notamment, comme on l'a vu, dans les analyses qu'il fait de la «présence civique » à Athènes, justement pour corriger Schmitt. Mais Meier, tout en saluant la référence, observe ensuite que Schmitt ne tient aucun compte de la cité grecque antique, de ce Dasein (pour employer plus nettement le vocabulaire heideggérien ici commun à Schmitt, Arendt et Meier) de la polis grecque. L'égalité des citoyens et le sens de la communauté ont disparu du concept schmittien :

Die griechische Bedeutung von 'politisch', also 'bürgerlich' (mit dem Akzent auf die politische Gleichheit der Bürger), 'im Sinne des Ganzen', 'gemeinsam', hat sich daraus in Schmitts Verständnis restlos verloren ${ }^{63}$.

La signification grecque de «politique », c'est-à-dire « civique » (avec l'accent mis sur l'égalité politique des citoyens), « dans le sens de la collectivité », « commun », a donc complètement disparu dans l'interprétation de Schmitt.

Si bien que Meier va jusqu’à parler d'une « usurpation » («ja, Usurpation ») par Schmitt du mot «politique », en se référant d'ailleurs à une

62. Sur H. Arendt et la philosophie politique grecque classique, je me permets de renvoyer à Demont 2002, où l'on trouvera une première bibliographie.

63. Meier 1988, p. 543. 
analyse comparable faite par Leo Strauss dès $1932^{64}$, et d'une façon beaucoup plus affirmée et polémique que dans son livre de $1980^{65}$. On comprend pourquoi il ne fait intervenir que de façon en quelque sorte accessoire, dans son analyse des Euménides, la question de l'alliance avec Argos et de la guerre extérieure : « Le concept d'ennemi ne joue absolument aucun rôle dans la plus grande part de la politique ${ }^{66}$. »

Christian Meier peut alors, dans une seconde partie, moyennant « l'inflexion » («Wendung ») qu'il fait subir à l'utilisation schmittienne de l'opposition ami/ennemi, expliquer sa propre tentative de définir le politique et résumer les analyses de son livre. Le sentiment de l'ennemi permet de fonder l'unité politique face à l'extérieur, mais le politique implique aussi l'effacement, la neutralisation, de ce sentiment à l'intérieur, entre les citoyens, si l'on étudie « l'histoire de la façon élémentaire dont les hommes se regroupent de façon politique (...) pour rendre possible protection, sécurité de l'existence et aussi, en particulier, décisions ${ }^{67}$, ce qui implique l'étude de la cité grecque, du politique comme citoyenneté, du pouvoir politique comme alternance et non comme domination. Or, même dans cette optique, maintient Christian Meier, « d'une façon très frappante », «les plus importantes (certes pas les plus extrêmes) conclusions de Carl Schmitt » se vérifient dans le premier texte sur le politique, selon lui, à savoir Les Euménides d'Eschyle ${ }^{68}$.

La fin de l'étude s'éloigne du politique grec, pour aborder en particulier le parcours qui a conduit Schmitt de la théorie de l'ami et de l'ennemi à l'antisémitisme ${ }^{69}$, et je la laisse donc de côté. On peut constater au moins un écart entre l'analyse que j'ai proposée et l'auto-analyse de Christian Meier : dans sa contribution au colloque sur Carl Schmitt, il ne mentionne la question de la «décision » qu'en passant, dans le passage que je viens de citer, alors que c'est un point de contact très important entre les deux auteurs, peut-être l'aspect le plus personnel de l'analyse des Euménides proposée par

64. Sur ce « dialogue entre absents », cf. H. Meier 1990.

65. « Je pense que la capacité de distinguer ami et ennemi n'est pas le critère du politique, mais seulement de l'unité politique (nur dasjenige der politischen Einheit). » (Meier 1988, p. 545.) Voir cependant déjà Meier 1995, p. 30-31.

66. Meier 1988, p. 547.

67. Ibid. p. 550 («die Geschichte der ganz elementaren Weise des politischen SichGruppierens von Menschen [...], um Schutz wie Daseinsfürsorge wie insbesondere auch Entscheidungen zu ermöglichen $\gg)$.

68. Ibid. p. 551.

69. Voir sur ce point, par exemple, Faye 2009. 
Meier, et que c'est un aspect essentiel de la théorie de $S \operatorname{chmitt}^{70}$. Il est vrai que c'est précisément le point le plus délicat de la référence, puisque l'inflexion proposée aboutit en fait à un renversement de la définition initiale, et que cela reste une aporie toujours actuelle de la pensée politique idéaliste, et peut-être de la pensée politique en général ${ }^{71}$.

Évoquons brièvement pour finir la façon dont le livre de Meier a été introduit dans la discussion publique en France, principalement par l'entremise de Paul Veyne, qui l'invita au Collège de France. Ce dernier préfaça ensuite, en 1984, le premier livre de Christian Meier publié en français, son Introduction à l'anthropologie politique de l'Antiquité classique ${ }^{72}$, issue de ses leçons au Collège de France, en le présentant comme étant à la fois un « historien » et un « politologue » construisant une histoire politique non événementielle, en somme comparable, dit-il, à la perspective de « notre École des Annales ». Paul Veyne laisse entendre que c'est lui qui a introduit dans le titre du livre la notion d'«anthropologie politique ». Ainsi, il rapprochait l'auteur du courant français de l'anthropologie de la Grèce antique, dont il est l'un des représentants les plus connus. Si Christian Meier lui-même, dans la postface de son second ouvrage publié en français, De la tragédie grecque comme art politique ${ }^{73}$, déclare avoir utilisé avec profit les travaux de «l'école de Jean-Pierre Vernant» sur «les structures profondes de la pensée grecque $\gg$, il ajoute qu'il trouve ses observations « trop largement axées sur des structures profondes, universelles », et qu'il veut «cerner de façon plus nette et plus rigoureuse le caractère original du corps civique athénien $\gg^{74}$. La construction de ce rapport à l'anthropologie telle qu'elle était pratiquée en France a fait l'objet d'une communication dans le volume d'études consacrées spécifiquement à l'œuvre de Christian Meier ${ }^{75}$. Une autre communication du même volume, consacrée à l'interprétation de la tragédie classique par Meier, discute avec

70. En un sens différent, Meier 2010 per litt.: «Es war vielerlei, was man bei C. S. lernen konnte. Einerseits eben zu Freund und Feind und zu Dezision, andererseits zu Politischen. 》

71. Le Professeur Meier m'écrit à ce propos: «Dieses 'Politische' hat mich sehr gequält. Ich hatte immer (und habe ich heute) das Gefühl, daß es ein wesentliches Thema war-sowie man es von C S. ausgehend konzipieren könnte. Aber ich bin eigentlich nie ganz zufrieden mit meinen Ausführungen darüber gewesen. » (Meier 2010 per litt.)

72. Meier 1984, p. 5-6.

73. Meier 1991, p. 269-271.

74. Réticences du même genre (et signalées par Martin 2008, p. 61) à l'égard des travaux de Sally Humphreys dans Meier 1995, p. 356-357 n. 17.

75. Martin 2008. 
une certaine précision l'éventuelle complémentarité entre ce dernier et les travaux de Jean-Pierre Vernant et de ses compagnons de lutte («mit JeanPierre Vernant und seinen 'Mitstreitern'») sur les rapports entre la politique et la tragédie, ainsi que les critiques de cette conception politique de la tragédie qui ont été formulées, entre autres, par Simon Goldhill ${ }^{76}$, et, ajoutons-le, par Nicole Loraux, dans une sorte de palinodie. Mais il semble bien que la réception française des travaux de Christian Meier sur «le politique » ait en partie reposé sur un malentendu autour de la notion même d'anthropologie, qui n'a pas le même sens en allemand, où elle désigne l'étude de l'homme en général et des «structures profondes » de son esprit, et en français, où le mot est employé, en un sens anglo-saxon, d'une façon assez comparable à l'emploi du mot «ethnologie ». En tout cas, elle n'a guère pris en compte sa tentative de légitimer d'un point de vue démocratique, en se fondant sur l'étude de l'Athènes classique, une partie des analyses de Carl Schmitt ${ }^{77}$.

76. Seidensticker 2008, notamment p. 75-78.

77. Sur le sens du mot en allemand, $c f$. par exemple Hose 2009, p. 7-8 : « Gründsätzlich will ich damit - in Abgrenzung zum angelsächsischen Gebrauch von 'anthropology', mit dem eine Ethnologie oder Völkerkunde gemeint zu werden pflegt, - gemäß der gängigen Auffassung im deutschen Sprachraum eine 'Wissenschaft vom Menschen' bezeichnen. » On trouvera une critique virulente du concept de «politique » dans Detienne 2005, p. 144-176 («Des comparables sur les balcons du politique ») : Carl Schmitt et sans doute Christian Meier y sont implicitement visés dans une allusion, p. 149, où M. Detienne dit ne pas vouloir « perdre de temps » à « inventorier » les analyses « de l'invention du politique » à partir de «la distinction de l'ami et de l'ennemi ». Marcel Detienne, lui, analyse des sociétés politiques variées selon une méthode comparative entièrement différente de celle de Christian Meier. Pour une autre critique, non moins virulente, adressée à l'usage conscient $\mathrm{du} \ll$ concept de politique » comme «mythe » (« instrument de combat contre le rationalisme politique, en particulier celui de la démocratie libérale où le politique se dégrade et se décompose en négociations et discussions », p. 10), $c f$. Zarka 2009b. On trouvera un état plus récent de la réflexion de Christian Meier sur le politique dans son étude «Polis und Staat. Zwei Ausprägungen des Politischen und die Frage nach seiner Zukunft » (Meier 2006), que je le remercie de m’avoir communiquée. 


\section{BIBLIOGRAPHIE}

BAUER, St. 2001 : Polisbild und Demokratieverständnis in Jacob Burckhardts Griechischer Kulturgeschichte, Bâle-Munich, 2001 (Beiträge zu Jacob Burckhardt, 3).

BernetT, M., W. NipPel \& A. WinTERLING (éd.) 2008 : Christian Meier zur Diskussion : Autorenkolloquium am Zentrum für Interdisziplinäre Forschung der Universität Bielefeld, Stuttgart, 2008.

BlumenberG, H. 1966 : Die Legitimität der Neuzeit, Francfort sur le Main, 1966. [Trad. fr. par M. Sagnol, J.-L. Schlegel et D. Trierweiler, avec la collab. de M. Dautrey, Paris, 1999.]

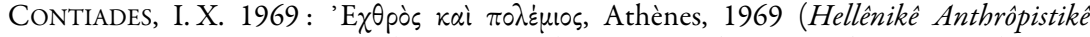
Hetaireia. Kentron Anthrôpistikôn Klassikôn Spoudôn. Seira deutera : Meletai kai ereunai, 19).

Demont, P. 2002: «Hannah Arendt et la philosophie politique grecque », dans: J. Leclant (présid.) et A. Michel (dir.), Tradition classique et modernité, Paris, 2002 (Cahiers de la Villa Kérylos, 13), p. 21-41.

-à paraître: «Y a-t-il une science du politique ? Les débats athéniens de l'époque classique », dans Actes du XVI congrès Guillaume Budé, L'homme et la science, Montpellier, $I^{e r}-4$ septembre 2008.

DetiEnNe, M. 2005 : Les Grecs et nous: une anthropologie comparée de la Grèce ancienne, Paris, 2005 (Pour l'histoire).

FAYE, J.-P. 2009 : « Carl Schmitt, Göring et l'“État total” » dans Zarka 2009, p. 161-181.

HOSE, M. 2009 : Euripides als Anthropologe, Munich, 2009 (Bayerische Akademie der Wissenschaften. Sitzungsberichte, 2009, 2).

JOUANNA, J. \& F. MONTANARI (éd.) 2009 : Eschyle à l'aube du théatre occidental: neuf exposés suivis de discussions, Vandouvres-Genève, 25-29 août 2008, Genève, 2009 (Entretiens sur l'Antiquité classique, 55).

Lomax, J.H. 2000 : « Carl Schmitt, Heinrich Meier, and the End of Philosophy », Interpretation, 28 (2000), p. 51-78.

MARTIN, J. 2008: «Zur Anthropologie des politischen Denkens », dans M. Bernett, W. Nippel \& A. Winterling, 2008, p. 61-69.

MeHrING, R. 2009: Carl Schmitt, Aufstieg und Fall : eine Biographie, Munich, 2009.

MeIER, Chr. 1973 : «Clisthène et le problème politique de la polis grecque », Revue internationale des droits de l'antiquité, 20 (1973), p. 115-159.

- 1980 : Die Entstehung des Politischen bei den Griechen, Frankfurt am Main, 1980.

- 1984: Introduction à l'anthropologie politique de l'Antiquité classique, traduit de l'allemand par P. Blanchard, Paris, 1984 (Collège de France. Essais et conférences).

- 1988: «Zu Carl Schmitts Begriffsbildung-Das Politische und der Nomos », dans H. Quaritsch (éd.), Complexio Oppositorum: über Carl Schmitt, Berlin, 1988, p. 537-556.

- 1991: De la tragédie grecque comme art politique, Paris, 1991 (Histoire, 9). [Traduction par M. Carlier de Die politische Kunst der griechischen Tragödie, Munich, 1988.]

- 1995: La naissance du politique, Paris, 1995 (NRF essais). [Traduction, par D. Trierweiler, de Meier 1980.]

- 2006: « Polis und Staat: zwei Ausprägungen des Politischen und die Frage nach seiner Zukunft », dans: H.Schmidinger \& Cl. Sedmak (éd.), Der Mensch : ein zôon politikón? Gemeinschaft - Öffentlichkeit-Macht, Darmstadt, 2006, p. 25-46.

- per litt. 2010 : Lettre manuscrite du Prof. Christian Meier (07/05). 
- 2010 : Compte rendu de R. Mehring 2009, Zeitschrift für Ideengeschichte, 4.3 (2010), p. 115-121.

MeIER, H. 1990 : Carl Schmitt, Leo Strauss et La notion de politique : un dialogue entre absents. Suivi du Commentaire de Leo Strauss sur « La Notion de politique » et de trois lettres inédites à Carl Schmitt des années 1932-1933, Paris, 1990 (Commentaire-Julliard). [Trad. fr. par F. Manent de Carl Schmitt, Leo Strauss und «Der Begriff des Politischen $\gg:$ zu einem Dialog unter Abwesenden, mit Leo Strauss' Aufsatz über den «Begriff des Politischen » und drei unveröffentlichten Briefen an Carl Schmitt aus den Jahren 1932-33, Stuttgart, 1988.]

Schмiт, C. 1932 : Der Begriff des Politischen, mit einer Rede über das Zeitalter der Neutralisierungen und Entpolitisierungen, Munich-Leipzig, 1932 (Wissenschaftliche Abhandlungen und Reden zur Philosophie, 10). [Rééd. : Der Begriff des Politischen, Text von 1932 mit einem Vorwort und drei Corollarien, Berlin, 1963.]

- 1963: Theorie des Partisanen: Zwischenbemerkung zum Begriff des Politischen, Berlin, 1963.

- 1972: La notion de politique; Théorie du partisan, Paris, 1972 (Liberté de l'esprit, 17). [Trad. par M.-L. Steinhauser de Schmitt 1932 et de Schmitt 1963.]

- 1988: Théologie politique: 1922, 1969, Paris, 1988 (Bibliothèque des sciences humaines). [Trad. fr. par J.-L. Schlegel de : Politische Theologie : 4 Kapitel zur Lehre von der Souveränität, Munich-Leipzig, 1922, et de Politische Theologie II: die Legende von der Erledigung jeder politischen Theologie, Berlin, 1970.]

- 1995 : Les trois types de pensée juridique, Paris, 1995 (Droit, éthique, société). [Trad. fr. par M. Köller \& D. Séglard, de Über die drei Arten des rechtswissenschaftlichen Denkens, Hambourg, 1934 (Schriften der Akademie für deutches Recht, 3).]

Seidensticker, B. 2008: «Tragödie und Polis»», dans M. Bernett, W. Nippel \& A. Winterling, 2008, p. 71-85.

Seve, M. 2006 : Aristote, Constitution d'Athènes: le régime politique des Athéniens, traduction nouvelle, introduction, notes et index, Paris, 2006 (Le livre de poche. 4688).

Sommerstein, A.H. 1989 : Aeschylus, Eumenides, edited with introduction and commentary, Cambridge, 1989 (Cambridge Greek and Latin Classics).

TRIERWEILER, D. 2009: « Georges Sorel et Carl Schmitt : d'une théorie politique du mythe à l'autre », dans Zarka 2009, p. 15-46.

TUCHSCHERER, E. 2004 : «Le Léviathan dans la doctrine de l'État de Thomas Hobbes: sens et échec du décisionnisme politique », Asterion [revue en ligne], 2 (2004).

ZARKA, Y. Ch. (éd.) 2009 : Carl Schmitt ou Le mythe du politique. Suivi de : Carl Schmitt, La théorie politique du mythe, trad. fr. par D. Trierweiler de Die politische Theorie des Mythos, 1923), Paris, 2009 (Débats philosophiques).

- 2009b: «Introduction: de la théorie politique du mythe à la mythologie politique », dans Zarka 2009, p. 9-13. 